\title{
Blaschke, Olaf/Rámon Solans, Francisco Javier (Hrsg.): Weltreligion im Umbruch. Transnationale Perspektiven auf das Christentum in der Globalisierung (Religion und Moderne, Bd. 12), 507 S., Campus, Frankfurt a. M./New York 2019.
}

\author{
Martin Lutz
}

Angenommen: 17. Dezember 2020 / Online publiziert: 5. Januar 2021

(C) Der/die Autor(en) 2021

Zwei Kernfragen stehen im Fokus des vorliegenden Sammelbands. Welchen Nutzen bringt ein globalhistorischer Zugang für die Religionsgeschichte und wie reagierte das Christentum auf die Globalisierungsprozesse im ,langen“19. Jahrhundert? Ausgangspunkt ist der Befund, dass die Religionsgeschichte des Christentums bislang ein auf den Nationalstaat bezogenes Untersuchungsfeld darstellt, für das Christentum seit dem 19. Jahrhundert aber Veränderungsprozesse durch Globalisierung und transnationale Beziehungen prägend waren. So lässt sich der Band auch als ein Produkt nachholender Entwicklung bezeichnen. Während andere historiografische Felder wie die Wirtschafts- und Migrationsgeschichte bereits seit zwei Jahrzehnten intensiv mit globalhistorischen Themen und Fragen arbeiten, sind diese für die Religionsgeschichte des Christentums noch weitgehend Neuland.

Der Band ist aus einer Tagung des Münsteraner Exzellenzclusters „Religion und Politik“ im Jahr 2016 entstanden und versammelt insgesamt 20 Beiträge. In seiner gründlichen und ausführlichen Einleitung weist der Mitherausgeber Olaf Blaschke darauf hin, dass für Katholizismus und Protestantismus kaum globalhistorische oder transnationale Studien vorliegen, mit Ausnahme von Arbeiten zur Missionsgeschichte. Umgekehrt habe aber ,,auch die Globalgeschichte das Thema Religion zugunsten von migrations- und wirtschaftshistorischen Themen eklatant ignoriert" (S. 17). Ziel des Bands ist es daher, den methodologischen Nationalismus in der Religionsforschung zu überwinden und das Thema anschlussfähig für breitere Diskurse zu machen.

Der Buchtitel lehnt sich folglich nicht zufällig an Thomas Nipperdeys „Religion im Umbruch“ an. Blaschke skizziert einführend den Umbruch von Religion unter Globalisierungsbedingungen, der nicht endogen aus dem Nationalstaat heraus er-

M. Lutz (四)

Humboldt-Universität zu Berlin, Berlin, Deutschland

E-Mail: Martin.Lutz@hu-berlin.de 
klärt werden könne. Die Einführung leistet eine gründliche Auseinandersetzung mit Globalgeschichte, insbesondere mit der zentralen - teils nicht mehr ganz neuen Grundlagenliteratur von Autoren wie Jürgen Osterhammel und Sebastian Conrad. Einschränkend ist hinzuzufügen, dass der Band nicht das Christentum als ganzes betrachtet, sondern auf Katholizismus und den - nahezu ausschließlich deutschen Protestantismus beschränkt ist. Die thematische Begrenzung ist an sich nachvollziehbar. Doch hätte eine kurze Diskussion der globalhistorischen Forschung zu anderen ,Weltreligionen' wie Islam und Judentum eine Einordnung des Forschungsfelds verbreitert. Auch innerhalb des christlichen Kosmos gibt es durchaus Beispiele anderer Religionsgemeinschaften wie die Quäker und Mennoniten, zu denen Studien in globalhistorischer und transnationaler Perspektive vorliegen (Goossen, Benjamin W.: Chosen Nation. Mennonites and Germany in a Global Era, 2017; Hadley, Herbert M.: Quakers World-Wide. A History of Friends World Committee for Consultation, 1991).

Der Band ist übersichtlich in zwei Teile gegliedert. Teil I beschäftigt sich in eher methodischer Perspektive mit der Nützlichkeit transnationaler und globalhistorischer Perspektiven auf das Christentum. Die Beiträge in Teil II gehen auf die Auswirkungen der Globalisierung auf das Christentum ein. Dieser Teil ist wiederum in zwei Abschnitte aufgeteilt. Im ersten sind Beiträge zusammengefasst, die sich mit Globalisierungsaspekten innerhalb des Protestantismus und Katholizismus beschäftigen. Programmatisch sind in der Abschnittsüberschrift mit „Diffusion oder Aneignung, Uniformierung oder Partikularisierung“ einige Begriffe vorgegeben, die die Bandbreite der Themen verdeutlichen. Der zweite Abschnitt fragt schließlich nach den Reaktionen auf Globalisierungsprozesse in Protestantismus und Katholizismus. Ob das Christentum in diesem Zusammenhang als „Opfer der Globalisierung“ (S. 30) bezeichnet werden kann, ist fraglich, scheint dieser Begriff doch problematisch zu sein. Klar wird allerdings, dass Globalisierungsprozesse auch zu Abwehrreaktionen seitens der christlichen Kirchen geführt haben.

Die Bandbreite der Themen ist überaus groß. Sie reichen zeitlich vom frühen 19. Jahrhundert bis in die 1920er Jahre. Prominent vertreten sind mehrere Beiträge zum katholischen Ultramontanismus; geografisch bildet Lateinamerika einen Schwerpunkt. Manche Aufsätze sind quellengesättigt und verdeutlichen, wie sich der globalhistorische Zugriff empirisch umsetzen lässt. Beispielsweise kann Frederik Schulze auf Basis von Reiseberichten und zeitgenössischen Publikationen sehr anschaulich die deutschnational geprägte protestantische Mission in Brasilien um 1900 als „globales Projekt“ (S. 379) aufzeigen. Andere Beiträge haben einen eher essayistischen Charakter und bieten keine empirischen Erkenntnisse. So ist der Aufsatz von Yvonne Maria Werner und Katharina Kunter eher als kritischer Literaturbericht mit einer Ideenskizze für ein Forschungsprogramm im Feld Gender, Religion und Globalgeschichte zu lesen.

Einen großen Mehrwert bieten die drei Kommentare zu den einzelnen Abschnitten von Detlef Pollack (,Was kann die Globalgeschichte von der Modernisierungstheorie lernen?“), Thomas Großbölting („Das Christentum als Agent der Globalisierung?“) und Volkhard Krech (,Globalisierung, Weltgesellschaft und Differenzierung“). Insbesondere die Religionssoziologen Pollack und Krech gehen dabei weit über eine Synthese der Beiträge hinaus und liefern weiterführende Impulse theoretischer Art. 
In beiden Kommentaren finden sich ausführliche Verweise auf eine systemtheoretische Interpretation von Religion und Globalisierung. Beide Autoren sind sich einig, dass sich die Geschichtswissenschaft allgemein und eine globalhistorisch angelegte Religionsforschung im Besonderen weitaus stärker als bisher auf theoretische Ansätze der Sozialwissenschaften beziehen sollten. Es wird mitunter eine deutliche Kritik an der bisherigen Historiografie geäußert. So habe die ,,additive Analyseform so mancher globalhistorischer Studien vor allem damit zu tun, dass das Konzept der Globalisierung kaum analytische Kriterien und theoretisch inspirierte Leitfragen aufweist und theoretisch weithin vage und unbestimmt bleibt“" (S. 180).

Man muss den unterbreiteten theoretischen Angeboten Pollacks (Modernisierungstheorie) und Krechs (Differenzierung und Weltgesellschaft) nicht im Einzelnen folgen, doch ihre Diagnose ist plausibel. Globalgeschichte und transnationale Beziehungen werden in dem Band (wie auch in der allgemeinen globalhistorischen Forschung) meist als Heuristiken verwendet, die zwar neue Perspektiven auf den Gegenstand ermöglichen, aber noch keine eigentliche Theoriebildung leisten. Freilich befindet sich die Globalgeschichte des Christentums, wie Blaschke nachdrücklich betont, erst am Beginn dieses Forschungsprozesses. Dafür bietet der Band eine hervorragende Ausgangsbasis.

Funding Open Access funding enabled and organized by Projekt DEAL.

Open Access Dieser Artikel wird unter der Creative Commons Namensnennung 4.0 International Lizenz veröffentlicht, welche die Nutzung, Vervielfältigung, Bearbeitung, Verbreitung und Wiedergabe in jeglichem Medium und Format erlaubt, sofern Sie den/die ursprünglichen Autor(en) und die Quelle ordnungsgemäß nennen, einen Link zur Creative Commons Lizenz beifügen und angeben, ob Änderungen vorgenommen wurden.

Die in diesem Artikel enthaltenen Bilder und sonstiges Drittmaterial unterliegen ebenfalls der genannten Creative Commons Lizenz, sofern sich aus der Abbildungslegende nichts anderes ergibt. Sofern das betreffende Material nicht unter der genannten Creative Commons Lizenz steht und die betreffende Handlung nicht nach gesetzlichen Vorschriften erlaubt ist, ist für die oben aufgeführten Weiterverwendungen des Materials die Einwilligung des jeweiligen Rechteinhabers einzuholen.

Weitere Details zur Lizenz entnehmen Sie bitte der Lizenzinformation auf http://creativecommons.org/ licenses/by/4.0/deed.de. 